\title{
Cross sections and isomeric cross-section ratios in the interactions of fast neutrons with isotopes of mercury
}

\author{
M. Al-Abyad, ${ }^{1,2}$ S. Sudár,,${ }^{1, *}$ M. N. H. Comsan, ${ }^{2}$ and S. M. Qaim ${ }^{1, \dagger}$ \\ ${ }^{1}$ Institut für Nuklearchemie, Forschungszentrum Jülich GmbH, D-52425 Jülich, Germany \\ ${ }^{2}$ Cyclotron Facility, Nuclear Research Center, Atomic Energy Authority, Cairo 13759, Egypt
}

(Received 8 September 2005; published 29 June 2006)

\begin{abstract}
Excitation functions were measured for the reactions ${ }^{196} \mathrm{Hg}(n, 2 n){ }^{195} \mathrm{Hg}^{m, g},{ }^{198} \mathrm{Hg}(n, 2 n){ }^{197} \mathrm{Hg}^{m, g}$, ${ }^{204} \mathrm{Hg}(n, 2 n){ }^{203} \mathrm{Hg},{ }^{198} \mathrm{Hg}(n, p){ }^{198} \mathrm{Au}^{g}$, and ${ }^{199} \mathrm{Hg}(n, p){ }^{199} \mathrm{Au}$ over the neutron energy range of 7.6-12.5 MeV. Quasimonoenergetic neutrons were produced via the ${ }^{2} \mathrm{H}(d, n)^{3} \mathrm{He}$ reaction using a deuterium gas target at the Jülich variable energy compact cyclotron CV 28. Use was made of the activation technique in combination with high-resolution, high-purity Ge detector $\gamma$-ray spectroscopy. All the data were measured for the first time over the investigated energy range. The transition from the present low-energy data to the literature data around 14 $\mathrm{MeV}$ is generally good. Nuclear model calculations using the codes STAPRE and EMPIRE-2.19, which employ the statistical and precompound model formalisms, were undertaken to describe the formation of both the isomeric and ground states of the products. The total reaction cross section of a particular channel is reproduced fairly well by the model calculations, with STAPRE giving slightly better results. Regarding the isomeric cross sections, the agreement between the experiment and theory is only in approximate terms. A description of the isomeric cross-section ratio by the model was possible only with a very low value of $\eta$, i.e., the $\Theta_{\text {eff }} / \Theta_{\text {rig }}$ ratio.
\end{abstract}

DOI: 10.1103/PhysRevC.73.064608

PACS number(s): 24.10.-i, 24.60.Dr, 25.10.+s, 25.40.-h

\section{INTRODUCTION}

Studies of excitation functions of neutron threshold reactions are of considerable importance in testing nuclear models as well as in practical applications. Furthermore, isomeric cross-section ratios are of fundamental significance. We chose to study the neutron-induced reactions on isotopes of mercury mainly for two reasons. First, mercury is being considered as a target material in spallation neutron sources. The $(n, x n)$ reaction cross sections of mercury isotopes are therefore important for calculations on neutron multiplication. Second, ${ }^{195} \mathrm{Hg}^{m}$ and ${ }^{197} \mathrm{Hg}^{m}$ are high-spin isomers (each having a value of $13 / 2^{-}$) as compared to the respective ground state (with spin $\left.1 / 2^{-}\right)$. The relative formation of those states in $(n, 2 n)$ reactions as a function of the incident neutron energy should thus be very interesting.

A literature survey showed that for the isotopes of mercury, almost no experimental information is available for neutroninduced reactions from their thresholds up to $12.5 \mathrm{MeV}$. Most of the data existing in the literature were measured at neutron energies around $14 \mathrm{MeV}$ [1-9]. The only exception is the ${ }^{198} \mathrm{Hg}(n, 2 n)^{197} \mathrm{Hg}^{m}$ reaction, where Király et al. [10] reported data for two neutron energies, namely, 11.2 and $12.5 \mathrm{MeV}$. The aim of this work was to determine the excitation functions of several neutron- induced reactions on isotopes of mercury near their thresholds and to compare them with the results of nuclear model calculations, with particular attention given to the formation of the isomeric states.

\footnotetext{
*Permanent address: Institute of Experimental Physics, University of Debrecen, H-4010 Debrecen, Hungary.

${ }^{\dagger}$ Corresponding address: s.m.qaim@fz-juelich.de
}

\section{EXPERIMENT}

Cross sections were measured by activation and identification of the radioactive products. This technique is very suitable for investigating low-yield reaction products and closely spaced low-lying isomeric states, provided their lifetimes are not too short. The details have been described over the years in many publications from Jülich [cf. Ref. 11-17]. Here we give some salient features relevant to the present measurements.

\section{A. Samples and irradiations}

About $5 \mathrm{~g}$ of $\mathrm{HgCl}_{2}$ powder of natural isotopic composition (99.9\% pure, provided by Chempur or ABCR GmbH, Germany) was pressed at $10 \mathrm{ton} / \mathrm{cm}^{2}$, and a pellet, $0.3 \mathrm{~cm}$ thick and $2.0 \mathrm{~cm}$ in diameter, was obtained. Several such pellets were prepared. Each pellet was placed in an aluminum capsule; the total thickness of each sample, including the capsule, was $0.62 \mathrm{~cm}$. Monitor foils of $\mathrm{Al}(200 \mu \mathrm{m}$ thick $)$ and $\mathrm{Fe}(100 \mu \mathrm{m}$ thick) of the same diameter as the capsule were then attached in front and at the back of each sample.

Irradiations were performed at the Jülich variable energy compact cyclotron CV 28. The quasimonoenergetic neutrons were produced via the ${ }^{2} \mathrm{H}(d, n)^{3} \mathrm{He}$ reaction $(Q=3.27 \mathrm{MeV})$ on a deuterium gas target $\left(3.7 \mathrm{~cm}\right.$ long, $1.8 \times 10^{5} \mathrm{~Pa}$ pressure). A sketch of the target is shown in Fig.1. The characteristics of this neutron source have been described earlier $[11,18,19]$. The samples were placed in the $0^{\circ}$ direction relative to the incident deuteron beam, at a distance of $1.0 \mathrm{~cm}$ from the beam stop. By changing the deuteron energy between 5.0 and $10.0 \mathrm{MeV}$, it was possible to obtain neutrons of energies between 7.5 and $12.5 \mathrm{MeV}$. The beam current was kept constant at 2-3 $\mu \mathrm{A}$. The time of irradiation varied between 5 and $6 \mathrm{~h}$. At each energy, two irradiations were made, one with the target 


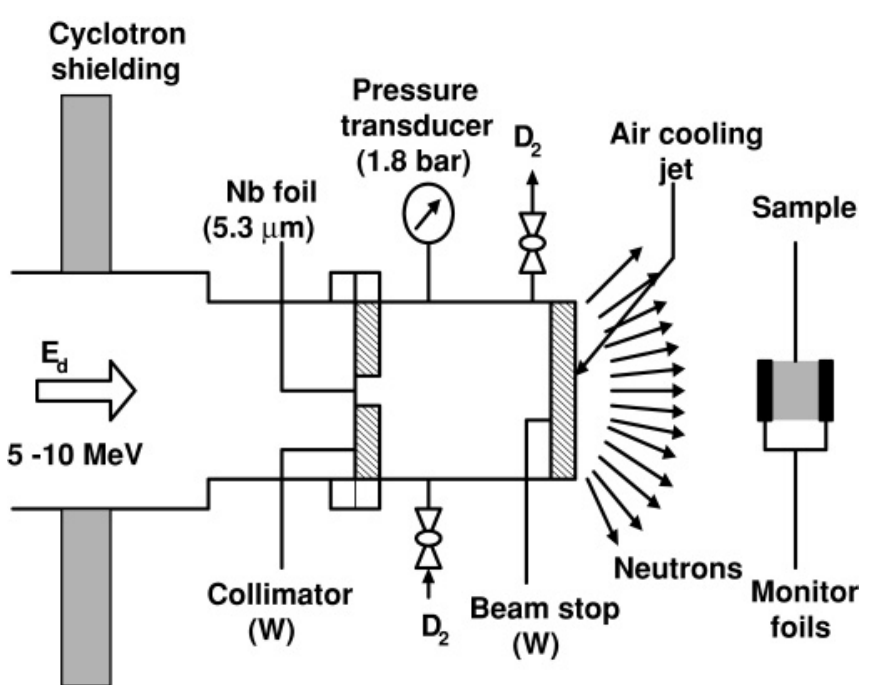

FIG. 1. Deuterium gas target used at the CV 28 cyclotron in Jülich to produce quasimonenergetic neutrons in the energy range of 7.5$12.5 \mathrm{MeV}$.

filled with the deuterium gas and the other as empty (gas in/gas out). This allowed a correction for the activity formed from the background neutrons.

The corrected activity $A_{\text {corr }}$ was calculated as

$$
A_{\mathrm{corr}}=A_{\mathrm{in}} C_{\mathrm{gas}} \text {, }
$$

where $A_{\text {in }}$ is the total activity. $C_{\text {gas }}$ is the background neutron correction determined via the gas-out measurement by the formula

$$
C_{\text {gas }}=1-\frac{A_{\text {out }} I_{\text {in }} m_{\text {in }}\left(1-e^{-\lambda t_{\text {in }}}\right)}{A_{\text {in }} I_{\text {out }} m_{\text {out }}\left(1-e^{-\lambda t_{\text {out }}}\right)},
$$

where "in" denotes the gas-in measurement, and "out" the gasout measurement. $A$ is the activity, $t$ the duration of irradiation, $m$ the sample mass, $I$ the beam current $(\mu A)$, and $\lambda$ the decay constant.

\section{B. Neutron energies and flux densities}

The average neutron energy effective at each sample was calculated using the Monte Carlo program NEUT [20,21] which takes into account the energy loss of the deuteron in the gas cell and the foils. The breakup of the deuteron on the deuterium gas was determined according to the results of Cabral et al. [22]. NEUT was also used to calculate the whole neutron spectrum which is divided into a breakup part and a monoenergetic part. The ratio of the activity induced by the monoenergetic neutrons to that by the breakup neutrons was calculated and used for the correction of the contribution of the breakup neutrons. The correction was of the order of a few percent, depending on the reaction threshold and the excitation function of the investigated reaction. An accurate measurement of the deuteron energy was performed completely on line; a personal computer controlled the position of the probe and evaluated the signal produced by the high frequency (HF) detector electronics [23]. The diameter of the beam falling on the front of the neutron target was $4 \mathrm{~mm}$. For ascertaining the constancy of the neutron flux, a constant check of the $\mathrm{D}_{2}$ gas pressure in the cell and of the deuteron beam current on the target was performed. The neutron flux density effective during each irradiation was determined via two monitor reactions, namely, ${ }^{56} \mathrm{Fe}(n, p){ }^{56} \mathrm{Mn}\left(T_{1 / 2}=2.58 \mathrm{~h}, E_{\gamma}=847 \mathrm{keV}, I_{\gamma}=\right.$ $98.9 \%)$, and ${ }^{27} \mathrm{Al}(n, \alpha){ }^{24} \mathrm{Na}\left(T_{1 / 2}=14.97 \mathrm{~h}, E_{\gamma}=1369\right.$ $\left.\mathrm{keV}, I_{\gamma}=100 \%\right)$. The cross sections of the monitor reactions were taken from the International Reactor Dosimetry File [24]. The flux densities were calculated after correction of monitor product activities from background neutrons. The average flux density effective on each sample was then obtained by taking the mean value of the calculated flux densities for the front and back foils.

\section{Measurement of radioactivity}

The activation products were identified by $\gamma$-ray counting and checking their half-lives. Table I gives the reactions investigated, their $Q$ values, and the decay data of the products $[25,26]$ used in the quantitative assay of the activity. For measurements, high-purity Ge detectors were used. The samples and monitor foils were placed either directly on the

TABLE I. Nuclear reactions studied and decay data of the products.

\begin{tabular}{lccccc}
\hline \hline Nuclear reaction & $\begin{array}{c}Q \text { Value } \\
(\mathrm{MeV})\end{array}$ & $\begin{array}{c}\text { Mode of } \\
\text { decay }(\%)\end{array}$ & Half-life & $E_{\gamma}(\mathrm{keV})$ & $I_{\gamma}(\%)$ \\
\hline${ }^{196} \mathrm{Hg}(n, 2 n){ }^{195} \mathrm{Hg}^{g}$ & -8.84 & $\mathrm{EC}(100)$ & $9.9 \mathrm{~h}$ & 779.8 & 7.0 \\
${ }^{196} \mathrm{Hg}(n, 2 n){ }^{195} \mathrm{Hg}^{m}$ & -9.05 & $\mathrm{IT}(54)$ & $41.6 \mathrm{~h}$ & 261.8 & 37.9 \\
& & $\mathrm{EC}(46)$ & & 560.3 & 7.5 \\
${ }^{198} \mathrm{Hg}(n, 2 n){ }^{197} \mathrm{Hg}^{g}$ & -8.49 & $\mathrm{EC}(100)$ & $64.14 \mathrm{~h}$ & 77.3 & 18.7 \\
& & & & 191.4 & 0.63 \\
${ }^{198} \mathrm{Hg}(n, 2 n){ }^{197} \mathrm{Hg}^{m}$ & -8.56 & $\mathrm{IT}(91.4)$ & $23.8 \mathrm{~h}$ & 134 & 33.0 \\
${ }^{204} \mathrm{Hg}(n, 2 n){ }^{203} \mathrm{Hg}^{198}$ & -7.50 & $\beta^{-}(100)$ & $46.6 \mathrm{~d}$ & 279.2 & 81.5 \\
${ }^{198} \mathrm{Hg}(n, p){ }^{198} \mathrm{Au}^{g}$ & -0.60 & $\beta^{-}(100)$ & $2.69 \mathrm{~d}$ & 41.8 & 96.0 \\
${ }^{199} \mathrm{Hg}(n, p){ }^{199} \mathrm{Au}$ & -5.48 & $\beta^{-}(100)$ & $3.13 \mathrm{~d}$ & 158.37 & 40.0 \\
& & & & 208.2 & 8.73 \\
\hline \hline
\end{tabular}


end cap of the detector or at a distance of $5 \mathrm{~cm}$. The peak area analysis was done using the software GAMMA VISION version 5.10, EG\&G, ORTEC.

The detector efficiency was determined experimentally using a selected set of $\gamma$-ray standard sources obtained from Amersham International and PTB Braunschweig. The count rate of each $\gamma$ ray was corrected for coincidence summing effect, geometric effect of the $\mathrm{HgCl}_{2}$ sample, and $\gamma$-ray selfattenuation in the sample. Furthermore, in order to determine the counting efficiency for the extended source placed directly on the end cap of the detector, a radioactive source especially prepared was used as in two earlier cases [27,28]. For this purpose, $10 \mathrm{mg}$ samples of $\mathrm{HgCl}_{2}$ and $\mathrm{Eu}_{2} \mathrm{O}_{3}$ were irradiated using a $14 \mathrm{MeV} d(\mathrm{Be})$ neutron source, and the induced activity in each sample was assayed via $\gamma$-ray spectrometry. The sample-to-detector distance was $10 \mathrm{~cm}$. Thereafter, the irradiated samples were thoroughly mixed with about $5 \mathrm{~g}$ of $\mathrm{HgCl}_{2}$ in a mortar. The mixture was then pressed to a pellet and placed in the Al capsule, as in the case of the sample for irradiation. A $\gamma$-ray spectrometric analysis of the prepared sample gave the effective efficiency of the detector for $\gamma$ rays of various energies, including self-attenuation.

\section{Corrections for interfering reactions}

Since in this work, mercury of natural isotopic composition was used as target material, it is likely that some of the studied activation products may be formed not only via the main routes mentioned but also via a few other interfering reactions. In the case of ${ }^{204} \mathrm{Hg}(n, 2 n){ }^{203} \mathrm{Hg}$ and ${ }^{198} \mathrm{Hg}(n, 2 n){ }^{197} \mathrm{Hg}^{m, g}$ reactions, for example, special care was necessary to correct for the effective background activity via the ${ }^{202} \mathrm{Hg}(n, \gamma){ }^{203} \mathrm{Hg}$ and ${ }^{196} \mathrm{Hg}(n, \gamma){ }^{197} \mathrm{Hg}^{m, g}$ processes, respectively. For this purpose, two procedures were applied. In the first method, the gas-out measurement gave the $(n, \gamma)$ contribution from the background neutrons of energies up to about $2 \mathrm{MeV}$. The $(n, \gamma)$ contribution from the faster neutrons was estimated using the calculated $d d$ neutron spectrum (from NEUT, see above) and the ${ }^{202} \mathrm{Hg}(n, \gamma)$ or ${ }^{196} \mathrm{Hg}(n, \gamma)$ excitation function reported in the literature [29]. Because of the low cross section of the $(n, \gamma)$ reaction over the energy range of 2 to $13 \mathrm{MeV}$ (decreasing from 10 to about $1 \mathrm{mb}$ ), the fast background neutron contribution to the formation of the activation product was much smaller than the slow neutron contribution. In the second method, irradiations were done also with neutrons of energies just below the thresholds of the two investigated $(n, 2 n)$ reactions. The product radioactivity was then assumed to be due to the disturbing $(n, \gamma)$ reaction. A further very small but slightly increasing contribution of the $(n, \gamma)$ process with increasing neutron energy was estimated via the calculational method described above. In general, the correction data from the two procedures agreed within a few percent. The final result was that the contribution of the ${ }^{202} \mathrm{Hg}(n, \gamma){ }^{203} \mathrm{Hg}$ process to the formation of ${ }^{203} \mathrm{Hg}$ was about $30 \%$; in the case of ${ }^{197} \mathrm{Hg}^{m, g}$, however, the contribution of the ${ }^{196} \mathrm{Hg}(n, \gamma){ }^{197} \mathrm{Hg}^{m, g}$ reaction turned out to be negligibly small $(<3 \%)$.

The other investigated cases with possible interferences were ${ }^{198} \operatorname{Hg}(n, p){ }^{198} \mathrm{Au}^{g}$ and ${ }^{199} \operatorname{Hg}(n, p){ }^{199} \mathrm{Au}$. The two activation products could also be formed via the ${ }^{199} \mathrm{Hg}(n, n p+d){ }^{198} \mathrm{Au}^{g}$ and ${ }^{200} \mathrm{Hg}(n, n p+d){ }^{199} \mathrm{Au}$ processes, respectively. A consideration of the $Q$ values of the latter two processes, however, suggested that their contributions to the investigated activation products ought to be small and within the limits of uncertainties of the $(n, p)$ reaction cross sections measured in this work. No corrections were therefore applied in those measurements.

\section{E. Calculation of cross sections and their uncertainties}

The count rates at the end of bombardment (EOB), after corrections for contributions from background neutrons, were converted to decay rates by introducing corrections for summing effects, $\gamma$-ray emission intensity and the efficiency of the detector. Cross sections were then calculated using the well-known activation equation.

The individual uncertainties in cross-section measurements using $d d$ neutrons have been described in several publications (cf. Refs. [11-17]). The uncertainty in the excitation function of the monitor reaction was taken as $4 \%$ and that in the averaging of the neutron flux as 5\%. The efficiency of the $\gamma$-ray detector (incorporating self-absorption, geometry, and pileup) had an uncertainty of about $6 \%$. The uncertainty in the decay data used was $<2 \%$. Besides these systematic uncertainties, the major random uncertainties involved were due to counting statistics $(2 \%-5 \%), \gamma$-ray peak area analysis $(2 \%-15 \%)$, and corrections for contributions from background neutrons $(2 \%-10 \%)$. Because of low count rates, the uncertainties in counting statistics and peak area analysis were relatively large in the low neutron energy region. In the high neutron energy range of 10-12 MeV, those two uncertainties were much lower, and the major source of uncertainty was the correction due to background neutrons. The total uncertainty in each cross-section value was obtained by combining all the individual uncertainties in quadrature; it amounted to about $20 \%$.

\section{F. Calculation of isomeric cross-section ratios}

The calculation of the experimental isomeric cross-section ratio $\sigma_{m} / \sigma_{g}$ for the isomeric pairs ${ }^{195} \mathrm{Hg}^{m, g}$ and ${ }^{197} \mathrm{Hg}^{m, g}$ was straightforward since both states decay independently, and their formation cross sections were also determined independently.

\section{NUCLEAR MODEL CALCULATIONS}

Cross sections were calculated using the codes STAPRE and EMPIRE (version 2.19). Some important details are given below.

\section{A. STAPRE calculations}

STAPRE [30] employs the Hauser-Feshbach formalism for the equilibrium emission and the exciton model for the preequilibrium (PE) emission. The transmission coefficients for neutrons, protons, deuterons, and $\alpha$ particles were provided as input data to the STAPRE code by means of the spherical optical code SCAT-2 [31] using global parameter sets. For neutrons, 
TABLE II. Activation cross sections determined in this work.

\begin{tabular}{|c|c|c|c|c|c|c|c|}
\hline \multirow[t]{2}{*}{$\left\langle E_{n}\right\rangle(\mathrm{MeV})$} & \multicolumn{7}{|c|}{ Cross section $(\mathrm{mb})$} \\
\hline & ${ }^{196} \mathrm{Hg}(n, 2 n){ }^{195} \mathrm{Hg}^{g}$ & ${ }^{196} \mathrm{Hg}(n, 2 n){ }^{195} \mathrm{Hg}^{m}$ & ${ }^{198} \mathrm{Hg}(n, 2 n)^{197} \mathrm{Hg}^{g}$ & ${ }^{198} \mathrm{Hg}(n, 2 n){ }^{197} \mathrm{Hg}^{m}$ & ${ }^{204} \mathrm{Hg}(n, 2 n){ }^{203} \mathrm{Hg}$ & $\left.{ }^{198} \mathrm{Hg}(n, p)\right)^{198} \mathrm{Au}^{g}$ & ${ }^{199} \mathrm{Hg}(n, p){ }^{199} \mathrm{Au}$ \\
\hline $7.66 \pm 0.22$ & & & & & $11.5 \pm 2$ & & \\
\hline $8.45 \pm 0.25$ & & & & & $263 \pm 48$ & & $0.13 \pm 0.02$ \\
\hline $9.73 \pm 0.28$ & $60 \pm 11$ & $161 \pm 30$ & $233 \pm 43$ & $128 \pm 23$ & $846 \pm 155$ & $0.2 \pm 0.04$ & $0.34 \pm 0.06$ \\
\hline $10.59 \pm 0.31$ & $385 \pm 71$ & $281 \pm 51$ & $500 \pm 92$ & $304 \pm 56$ & $1071 \pm 197$ & $0.43 \pm 0.07$ & $0.75 \pm 0.10$ \\
\hline $11.07 \pm 0.34$ & $444 \pm 81$ & $385 \pm 70$ & $655 \pm 120$ & $405 \pm 74$ & $1285 \pm 236$ & $0.6 \pm 0.10$ & $1.0 \pm 0.20$ \\
\hline $11.25 \pm 0.34$ & $554 \pm 102$ & $507 \pm 93$ & $848 \pm 156$ & $400 \pm 73$ & $1584 \pm 291$ & $0.7 \pm 0.10$ & $1.3 \pm 0.20$ \\
\hline $11.51 \pm 0.34$ & $662 \pm 121$ & $591 \pm 108$ & $692 \pm 127$ & $518 \pm 95$ & $1665 \pm 306$ & $1.1 \pm 0.20$ & $1.6 \pm 0.30$ \\
\hline $11.92 \pm 0.35$ & $780 \pm 143$ & $570 \pm 104$ & $811 \pm 149$ & $535 \pm 98$ & $1495 \pm 275$ & $1.2 \pm 0.20$ & $1.6 \pm 0.30$ \\
\hline $12.40 \pm 0.36$ & $786 \pm 144$ & $862 \pm 158$ & $870 \pm 160$ & $540 \pm 99$ & $1721 \pm 316$ & $1.9 \pm 0.30$ & $2.3 \pm 0.42$ \\
\hline $12.53 \pm 0.50$ & $923 \pm 170$ & $990 \pm 182$ & $955 \pm 175$ & $667 \pm 122$ & $1903 \pm 350$ & $2.3 \pm 0.40$ & $1.9 \pm 0.35$ \\
\hline
\end{tabular}

the optical model parameter set of [32] and for protons that of [33] were used. In the case of $\alpha$ particles, a modified set of optical model parameters of [34] was used. For the energy $(E)$ and mass dependence $(A)$ of the effective matrix element $(M)$ of the internal transition, the $|M|^{2}=(F M) A^{-3} E^{-1}$ formula was applied, with the value of $F M=535 \mathrm{MeV}^{3}$. The energies, spins, parities, and branching ratios of the discrete levels were obtained by using the National Nuclear Data Center's Evaluated Nuclear Structure Data File (ENSDF) [26]. In cases where the spin and parity were not known, estimates from adjacent levels were made. In the continuum region, the level density was calculated by the back-shifted formula and the level density parameter given in Refs. [35,36]. The level density parameter $a$ for the calculation was selected by interpolating the data of the neighboring isotopes, taking into account the odd-even systematics. The back-shift parameter $\Delta$ was determined individually for all nuclei used in the model calculation. The cumulative plot of the known discrete levels, collected from the ENSDF, was fitted by the backshifted Fermi gas (BSFG) formula, while the level spacing at the neutron binding energy was kept according to the experimental value. The spin distribution of the level density $\eta$ was characterized by the ratio of the effective moment of inertia $\Theta_{\text {eff }}$ to the rigid-body moment of inertia $\Theta_{\text {rig }}$ (i.e., $\eta \Theta_{\text {eff }} / \Theta_{\text {rig }}$ ), and the calculations were performed for different $\eta$ values to find the best agreement with the experimental data. The transmission coefficients of photons are also of considerable significance in calculations on isomeric cross sections. They were derived from the $\gamma$-ray strength functions. For the $E 1$ transition, the Brink-Axel model with global parameters was applied; while for the $M 1, E 2, M 2, E 3$, and $M 3$ radiation, the Weisskopf model was used.

\section{B. EMPIRE calculations}

EMPIRE (version 2.19) [37] also makes use of the HauserFeshbach and the exciton model formalisms. Furthermore, it combines several other modern features described below. In these calculations, the standard library of input parameters was used which includes the nuclear masses, optical model parameters, ground state deformations, discrete levels and decay schemes, level densities, moments of inertia (MOMFIT), and strength function. The direct contribution was determined via the coupled channel calculation using the built-in ECISO3 code. The particle transmission coefficients were generated via the spherical optical model using the computer code ECIS03 and the default set of global parameters: for neutrons and protons from [38], and for $\alpha$ particles from [34]. In the calculation, the multi-step direct, multi-step compound, Hauser-Feshbach model with width fluctuation correction (HRTW), the DEGAS and PCROSS codes were used. These codes conserve the particle flux by dividing the absorption cross section of the optical model between the different types of reaction mechanisms. For the level densities, the Hartree-Fock (HF)-BCS microscopic level densities were used.

\section{RESULTS AND DISCUSSION}

\section{A. Cross sections and excitation functions}

The cross sections measured over the energy range of 7.6 to 12.5 MeV are given in Table II. Except for two data points at 11.2 and $12.5 \mathrm{MeV}$ for the ${ }^{198} \mathrm{Hg}(n, 2 n){ }^{197} \mathrm{Hg}^{m}$ reaction [10], all the data reported in this work in the studied energy range have been determined for the first time. The various reactions investigated are discussed below.

$$
\text { 1. }{ }^{196} \mathrm{Hg}(n, 2 n){ }^{195} \mathrm{Hg}^{m, g}
$$

The irradiated sample was counted immediately after EOB to determine the independent formation cross section of ${ }^{195} \mathrm{Hg}^{g}$ $\left(T_{1 / 2}=9.9 \mathrm{~h}\right)$. After a decay time of about $50 \mathrm{~h}$, it was counted again to measure the activity of ${ }^{195} \mathrm{Hg}^{m}\left(T_{1 / 2}=\right.$ 41.6 h). The present cross-section data are shown in Figs. 2 and 3 as a function of neutron energy, together with the literature data around $14 \mathrm{MeV}$ [4,5,7]. The transition from

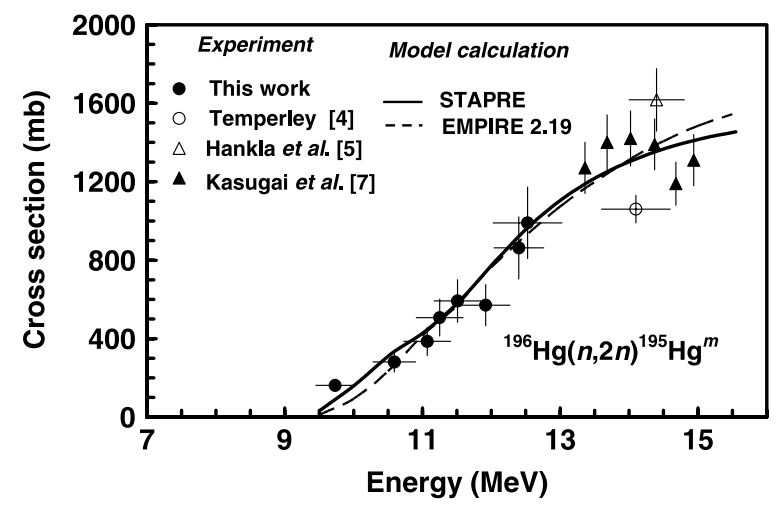

FIG. 2. Excitation function of the ${ }^{196} \mathrm{Hg}(n, 2 n){ }^{195} \mathrm{Hg}^{m}$ reaction. 


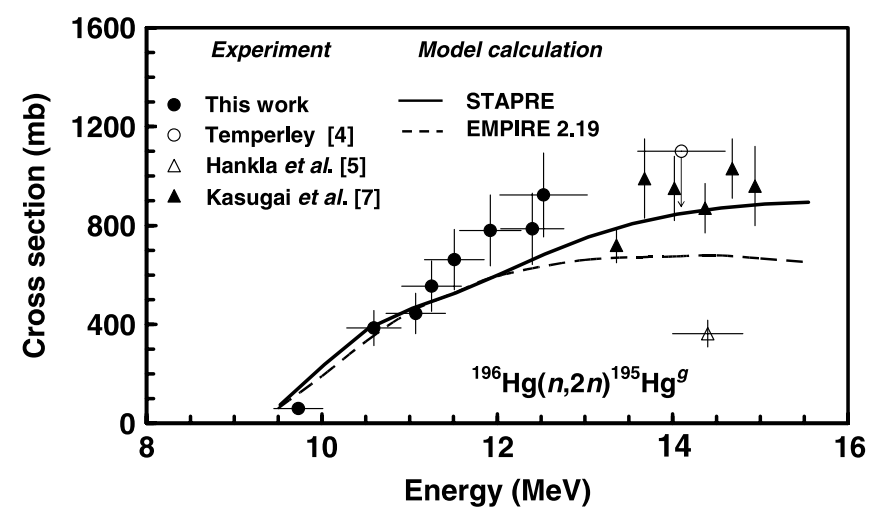

FIG. 3. Excitation function of the ${ }^{196} \mathrm{Hg}(n, 2 n){ }^{195} \mathrm{Hg}^{g}$ reaction.

the low-energy region to the higher one is smooth; only a few data points in the $14 \mathrm{MeV}$ region do not fit into the general trend (cf. the value for ${ }^{195} \mathrm{Hg}^{m}$ by Temperley [4] and the one for ${ }^{195} \mathrm{Hg}^{g}$ by Hankla et al. [5]). The results of the two nuclear model calculations, STAPRE and EMPIRE 2.19 , reproduce the experimental excitation function of the ${ }^{196} \mathrm{Hg}(n, 2 n){ }^{195} \mathrm{Hg}^{m}$ reaction very well. In the case of the ${ }^{196} \mathrm{Hg}(n, 2 n){ }^{195} \mathrm{Hg}^{g}$ reaction, whereas the STAPRE calculation reproduces the experimental excitation function very well, the EMPIRE produces rather low results in the energy range above 12.5 MeV.

$$
\text { 2. }{ }^{198} \mathrm{Hg}(n, 2 n){ }^{197} \mathrm{Hg}^{m, g}
$$

The first measurement in this case was also done immediately after EOB to determine the independent formation cross section of ${ }^{197} \mathrm{Hg}^{m}\left(T_{1 / 2}=23.8 \mathrm{~h}\right)$. Thereafter, measurement was done about one week after EOB to determine the activity of ${ }^{197} \mathrm{Hg}^{g}\left(T_{1 / 2}=64.1 \mathrm{~h}\right)$; the contribution via the decay of ${ }^{197} \mathrm{Hg}^{m}$ was then subtracted.

The present results are shown in Figs. 4 and 5 together with the literature data $[4-7,10]$, mainly in the energy region around $14 \mathrm{MeV}$. Again, the transition from the low- to highenergy region is smooth; however, our results for ${ }^{197} \mathrm{Hg}^{m}$ are considerably lower than the data by Király et al. [10]. The results of the two nuclear model calculations differ appreciably from each other in some energy regions. For ${ }^{197} \mathrm{Hg}^{m}$, in the low-energy region, the EMPIRE calculation appears to be in better agreement with our data. In the case of ${ }^{197} \mathrm{Hg}^{g}$, near

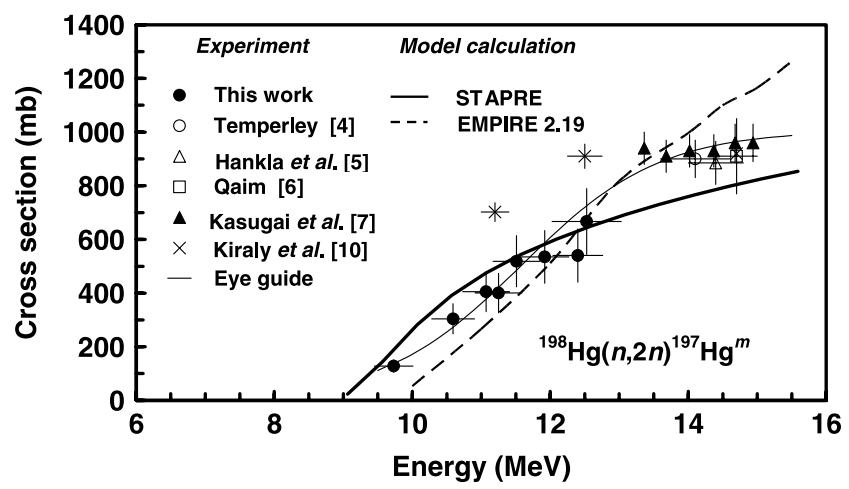

FIG. 4. Excitation function of the ${ }^{198} \mathrm{Hg}(n, 2 n){ }^{197} \mathrm{Hg}^{m}$ reaction.

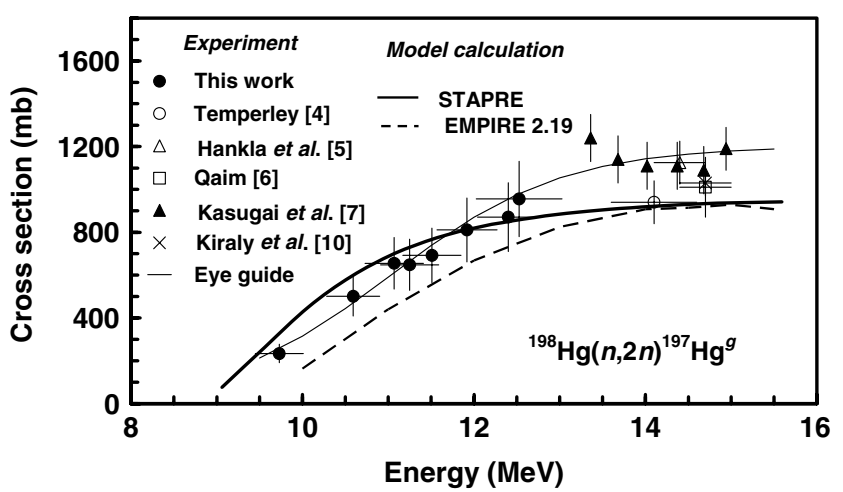

FIG. 5. Excitation function of the ${ }^{198} \mathrm{Hg}(n, 2 n){ }^{197} \mathrm{Hg}^{g}$ reaction.

the threshold, the STAPRE results are somewhat higher than the experimental data and the EMPIRE results are lower.

$$
\text { 3. }{ }^{204} \mathrm{Hg}(n, 2 n)^{203} \mathrm{Hg}
$$

In the study of this reaction, a subtraction of the ${ }^{202} \mathrm{Hg}(n, \gamma){ }^{203} \mathrm{Hg}$ contribution was necessary. We determined this contribution by a careful analysis as described above. Furthermore, the measurement of the sample was started about one week after EOB and continued for several weeks. This was necessary to avoid disturbance from the $23.8 \mathrm{~h}^{197} \mathrm{Hg}^{m}$ which emits a $\gamma$ ray in the vicinity of the $279 \mathrm{keV} \gamma$ ray used for the assay of ${ }^{203} \mathrm{Hg}$.

The results obtained are shown in Fig. 6 together with the literature data in the energy range of 13-18 MeV [1-7]. Again, the transition from the low- to high-energy region is smooth. The database seems to be now established. This is an important reaction from the viewpoint of neutron multiplication in a spallation source using mercury as target material. The results of nuclear model calculations reproduce the experimental data rather well, except for the region around the maximum of the excitation function. In particular, the EMPIRE results are too high.

$$
\text { 4. }{ }^{198} \mathrm{Hg}(n, p){ }^{198} \mathrm{Au}^{g}
$$

The cross-section data for the independent formation of ${ }^{198} \mathrm{Au}^{g}$ are shown in Fig. 7. The transition from our data to higher energy data $[4,5,7]$ is good, and the results appear

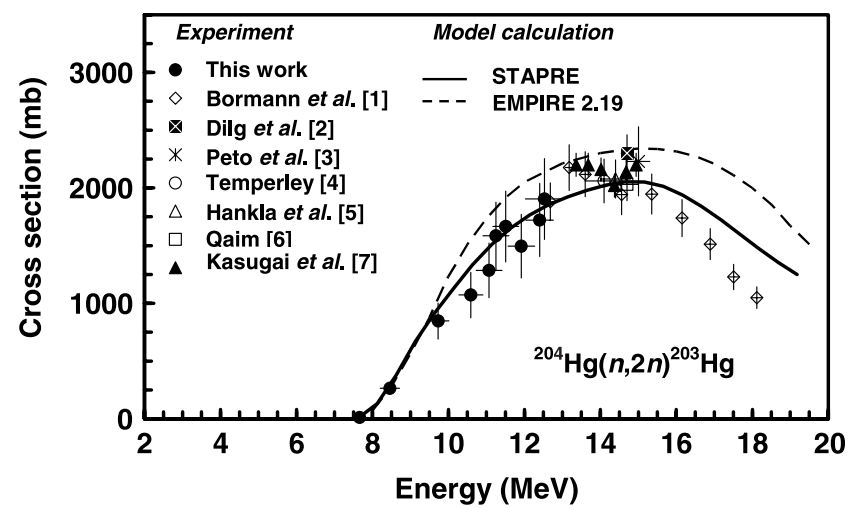

FIG. 6. Excitation function of the ${ }^{204} \mathrm{Hg}(n, 2 n)^{203} \mathrm{Hg}$ reaction. 


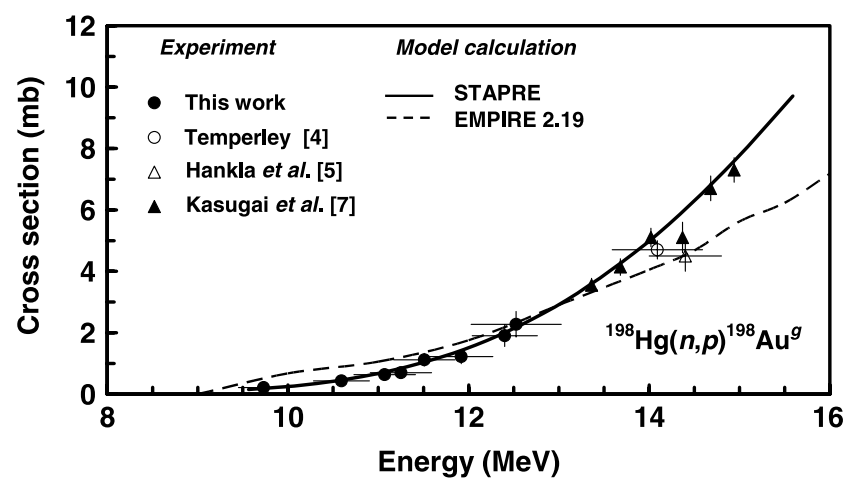

FIG. 7. Excitation function of the ${ }^{198} \mathrm{Hg}(n, p){ }^{198} \mathrm{Au}^{g}$ reaction.

to be consistent. Both the model calculations reproduce the cross sections fairly well up to about $14.5 \mathrm{MeV}$. At higher energies, the EMPIRE results appear to be somewhat low. For the isomer production (i.e., ${ }^{198} \mathrm{Hg}(n, p){ }^{198} \mathrm{Au}^{m} ; T_{1 / 2}=2.27 \mathrm{~d}$ ), we could not measure the cross section because no $\gamma$-ray peak corresponding to the decay of ${ }^{198} \mathrm{Au}^{m}$ was observed.

$$
\text { 5. }{ }^{199} \mathrm{Hg}(n, p){ }^{199} \mathrm{Au}
$$

The measured cross sections for this reaction are shown in Fig. 8. The present data and the literature data $[4,7]$ give a smooth excitation function; the only exception is the one data point by Hankla et al. [5] which is too low. The results of both the model calculations are in fairly good agreement with the experimental data, though the EMPIRE values for neutron energies above $13 \mathrm{MeV}$ are somewhat low.

\section{B. Isomeric cross-section ratios}

The experimental results on the isomeric crosssection ratios $\left(\sigma_{m} / \sigma_{g}\right)$ for the isomeric pairs formed in ${ }^{196} \mathrm{Hg}(n, 2 n){ }^{195} \mathrm{Hg}^{m, g}$ and ${ }^{198} \mathrm{Hg}(n, 2 n){ }^{197} \mathrm{Hg}^{m, g}$ reactions are given in Figs. 9 and 10, respectively. The data up to $12.5 \mathrm{MeV}$ are from this work, and those around $14 \mathrm{MeV}$ from the literature $[4-7,10]$. The ratio for both the pairs has a value of about 0.6 near the reaction threshold, depicting that at low excitation energies the low-spin isomer is preferentially populated. With the increasing incident neutron energy the ratio increases, showing that at higher excitation energies the formation of

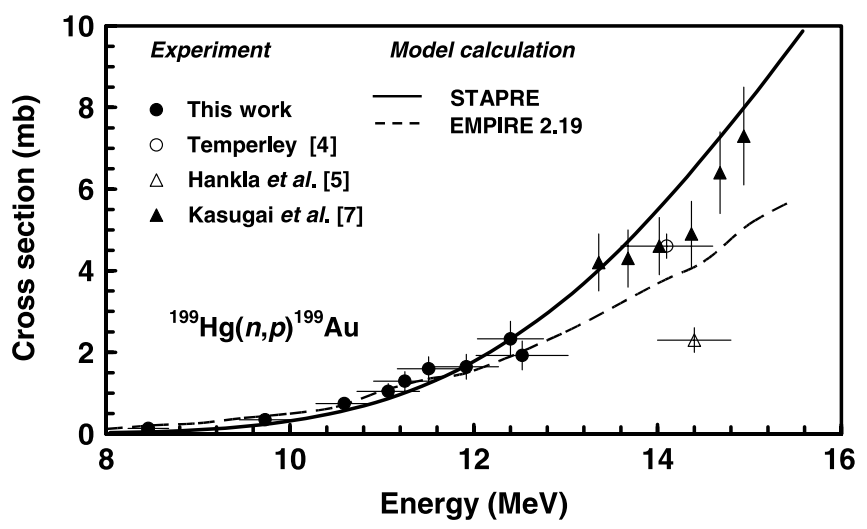

FIG. 8. Excitation function of the ${ }^{199} \mathrm{Hg}(n, p){ }^{199} \mathrm{Au}$ reaction.

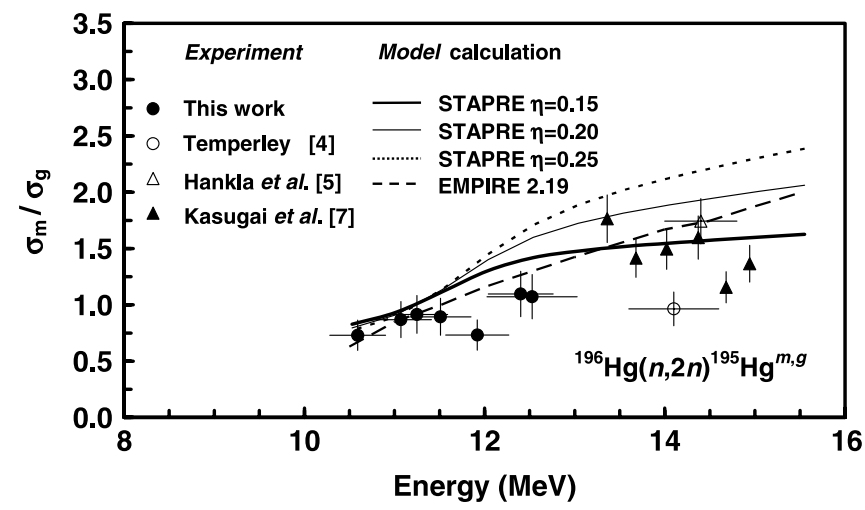

FIG. 9. Isomeric cross-section ratio for the pair ${ }^{195} \mathrm{Hg}^{m, g}$ in the ${ }^{196} \mathrm{Hg}(n, 2 n)$ reaction shown as a function of the incident neutron energy. The STAPRE calculation is shown for different values of $\eta$.

the high-spin isomer is more favored. This trend is similar to that for several other neutron- and charged-particle-induced reactions near their thresholds [16,17,39-44]. The increase seems to be more pronounced for ${ }^{195} \mathrm{Hg}^{m, g}$ than for ${ }^{197} \mathrm{Hg}^{m, g}$. At the highest neutron energy of about $15 \mathrm{MeV}$, the ratio for ${ }^{195} \mathrm{Hg}^{m, g}$ is about 1.5 whereas that for ${ }^{197} \mathrm{Hg}^{m, g}$ it is only 0.75 .

The results of the two nuclear model calculations are also given in Figs. 9 and 10. There appears to be good agreement between the experiment and theory, though in the energy region above about $13.5 \mathrm{MeV}$ the EMPIRE code tends to overestimate the isomeric cross-section ratio. Worth noting is that not only isomeric pairs with small differences in spins can be described by the model calculations (cf., for example, Refs. [17,3944]) but also the pairs with relatively large spin differences (as in this work), provided the input parameters are properly chosen. In this regard, two factors need special consideration: first, the input level scheme up to the continuum, and second, the spin distribution of the level density $(\eta)$. As mentioned above, we used the latest information available on the level schemes. Regarding the spin distribution of the level density, we performed STAPRE calculations using three values of $\eta$, namely, $0.15,0.20$, and 0.25 , for the pair ${ }^{195} \mathrm{Hg}^{m, g}$, and the results are given in Fig. 9. The value 0.15 appears to give better results. For the pair ${ }^{197} \mathrm{Hg}^{m, g}$ calculations were done

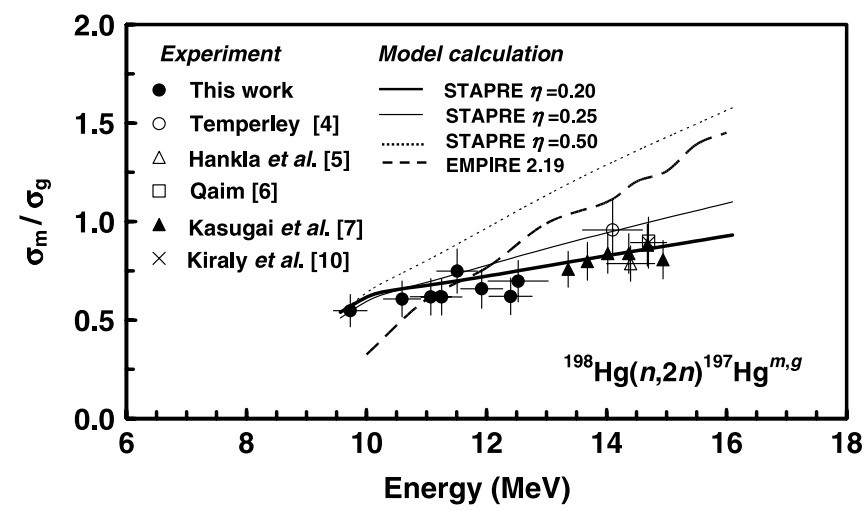

FIG. 10. Isomeric cross-section ratio for the pair ${ }^{197} \mathrm{Hg}^{m, g}$ in the ${ }^{198} \mathrm{Hg}(n, 2 n)$ reaction shown as a function of the incident neutron energy. The STAPRE calculation is shown for different values of $\eta$. 
with $\eta$ values of $0.20,0.25$, and 0.50 , and the results are given in Fig. 10. The value of 0.25 gives the best results.

It is worth pointing out that in the light and medium mass nuclei the isomeric cross-section ratio seems to be better reproduced by the $\eta$ values between 0.5 and 1.0 (cf. Refs. $[39,41,42,44])$. In the present work dealing with mercury isotopes, better results are obtained using a relatively low $\eta$ value $(<0.25)$. We recently came to the same conclusion from a study of the isomeric pairs ${ }^{195} \mathrm{Hg}^{m, g}$ and ${ }^{197} \mathrm{Hg}^{m, g}$ formed in ${ }^{3} \mathrm{He}$ - and $\alpha$-particle-induced reactions [45]. The relatively low value of $\eta$ describing the isomeric cross-section ratio in both neutron- and charged-particle-induced reactions confirms our postulate that $\eta$ is mass dependent. The energy of the incident neutrons was relatively low compared with those in the case of the charged-particle-induced reactions [45], and therefore the excitation energy of the compound nucleus in the present work was relatively low. Nevertheless, the dependence of the isomeric cross-section ratio on the parameter $\eta$ is still evident. The conclusion that $\eta$ is mass dependent is somewhat surprising. It may, however, inspire more theoretical work on the spin distribution of the level density.

\section{CONCLUSION}

Experimental results reported in this work should provide a good database for the $(n, 2 n)$ and $(n, p)$ reactions on several isotopes of mercury, especially with regard to calculations of neutron multiplication while using mercury as a target material in a spallation neutron source. Nuclear model calculations using the codes STAPRE and EMPIRE 2.19 showed that the total reaction cross section of a particular channel under consideration is reproduced fairly well by the Hauser-Feshbach formalism including precompound effects, with STAPRE giving slightly better results. In the case of the isomeric cross section, however, the agreement between the experiment and theory is only in approximate terms. The same is true for the isomeric cross-section ratio. Despite the relatively high spins of the metastable states, the isomeric cross-section ratios are relatively high for the pairs ${ }^{195} \mathrm{Hg}^{m, g}$ and ${ }^{197} \mathrm{Hg}^{m, g}$ even at low excitation energies; they increase with the increasing projectile energy. The agreement between the experimental data and the STAPRE calculation is good when $\eta=0.25$ or less is used.

\section{ACKNOWLEDGMENTS}

We thank Prof. H. H. Coenen for his support of this work, the crew of the compact cyclotron CV 28 at Jülich for performing the irradiations, and S. Spellerberg for experimental assistance. This work was done under a German-Egyptian bilateral research cooperation, and we are grateful to the concerned authorities in both countries for their support.
[1] M. Bormann, H.-K. Feddersen, H.-H. Holscher, W. Scobel, and H. Wagner, Z. Phys. A 277, 203 (1976).

[2] W. Dilg, H. Vonach, G. Winkler, and P. Hille, Nucl. Phys. A118, 9 (1968).

[3] G. Petö, P. Bornemisza-Pauspertl, and J. Karolyi, Acta Phys. Hung. 25, 91 (1968).

[4] J. K. Temperley, Phys. Rev. 178, 1904 (1969).

[5] A. K. Hankla, R. W. Fink, and J. H. Hamilton, Nucl. Phys. A180, 157 (1972).

[6] S. M. Qaim, Nucl. Phys. A185, 614 (1972).

[7] Y. Kasugai, F. Maekawa, Y. Ikeda, and H. Takeuchi, J. Nucl. Sci. Technol. 38, 1048 (2001).

[8] EXFOR, Nuclear reaction data, accessed on line at http://www. nndc.bnl.gov/nndc/exf or www.nea.frorwww-nds.iaea.or.at/ exfor.

[9] CINDA-A, The Index to Literature and Computer Files on Microscopic Neutron Data, IAEA, Vienna, 1990; CINDA 2000,The Index to Literature and Computer Files on Microscopic Neutron Data, IAEA, Vienna, 2003.

[10] B. Király, J. Csikai, and R. Doczi, in Proceedings of the 2000 Symposium on Nuclear Data, November 16-17, 2000, JAERI, Tokai, Japan, JAERI-Conf. 2001-006, INDC (JPN)188/4 (2001)283, 2001.

[11] S. M. Qaim, R. Wölfle, M. M. Rahman, and H. Ollig, Nucl. Sci. Eng. 88, 143 (1984).

[12] M. M. Rahman and S. M. Qaim, Nucl. Phys. A435, 43 (1985).

[13] M. Ibn-Majah and S. M. Qaim, Nucl. Sci. Eng. 104, 271 (1990).

[14] I.-G. Birn and S. M. Qaim, Nucl. Sci. Eng. 116, 125 (1994).
[15] M. Bostan and S. M. Qaim, Phys. Rev. C 49, 266 (1994).

[16] F. Cserpák, S. Sudár, J. Csikai, and S. M. Qaim, Phys. Rev. C 49, 1525 (1994).

[17] C. D. Nesaraja, S. Sudár, and S. M. Qaim, Phys. Rev. C 68, 024603 (2003).

[18] A. Grallert, J. Csikai, S. M. Qaim, and J. Knieper, Nucl. Instrum. Methods Phys. Res. A 334, 154 (1993).

[19] A. Grallert, J. Csikai, and S. M. Qaim, Nucl. Instrum. Methods Phys. Res. A 337, 615 (1994).

[20] I.-G. Birn, Kernforschungsanlage Jülich, Internal Report No. INC-IB-1, 1992.

[21] I.-G. Birn, CEC-JRC, IRMM, Geel, Internal Report No. $\mathrm{GE} / \mathrm{R} / \mathrm{VG} / 85 / 94,1994$.

[22] S. Cabral, B. Börker, H. Klein, and W. Mannhart, Nucl. Sci. Eng. 106, 308 (1990).

[23] Z. Kormány, Nucl. Instrum. Methods Phys. Res. A 337, 258 (1994).

[24] International Reactor Dosimetry File, IRDF-2002, available online at http://www-nds.iaea.org/irdf2002.

[25] R. B. Firestone and V. S. Shirley, Table of Isotopes (John Wiley \& Sons, New York, 1996).

[26] Evaluated Nuclear Structure Data File (ENSDF), database version of December 21, 2005, available online http://nndc.bnl.gov/ensdf/.

[27] R. M. Klopries, R. Doczi, S. Sudár, J. Csikai, and S. M. Qaim, Radiochim. Acta 76, 3 (1997).

[28] C. Nesaraja, K.-H. Linse, S. Spellerberg, S. Sudar, A. Suhaimi, and S. M. Qaim, Radiochim. Acta 86, 1 (1999).

[29] J. Kopecky, Report INDC(NDS)-362, IAEA, Vienna, 1997. 
[30] M. Uhl and B. Strohmaier, Computer code for particle induced activation cross section and related quantities, Institut für Radiumforschung und Kernphysik, Vienna, 1976, Report IRK $76 / 01$.

[31] O. Bersillon, Un programme de modèle optique spherique, Centre d'Etudes de Bruyéres-le Châtel, Paris, 1981, Report CEA-N2227.

[32] J. C. Ferrer, J. D. Carlson, and J. Rapaport, Nucl. Phys. A275, 125 (1977).

[33] F. G. Perey, Phys. Rev. 131, 745 (1962).

[34] L. McFadden and G. R. Satchler, Nucl. Phys. 84, 177 (1966).

[35] W. Dilg, W. Schantl, H. Vonach, and M. Uhl, Nucl. Phys. A217, 269 (1973).

[36] V. Plyaskin and R. Kosilov, Selected articles translated from Jadernye Konstanty (Nuclear Constants), INDC(CCP)-424, p. 27, IAEA, Vienna, 2000.
[37] M. Herman, R. Capote, B. Carlson, P. Oblozinsky, M. Sin, A. Trakov, and V. Zerkin, EMPIRE-II, Nuclear reaction model code, version 2.19, IAEA, Vienna, 2005.

[38] A. J. Koning and J. P. Delaroche, Nucl. Phys. A713, 231 (2003).

[39] S. M. Qaim, A. Mushtaq, and M. Uhl, Phys. Rev. C 38, 645 (1988).

[40] S. M. Qaim, M. Ibn Majah, R. Wölfle, and B. Strohmaier, Phys. Rev. C 42, 363 (1990).

[41] N. I. Molla, S. M. Qaim, and M. Uhl, Phys. Rev. C 42, 1540 (1990).

[42] S. Sudár, F. Szelecsényi, and S. M. Qaim, Phys. Rev. C 48, 3115 (1993).

[43] I.-G. Birn, B. Strohmaier, H. Freiesleben, and S. M. Qaim, Phys. Rev. C 52, 2546 (1995).

[44] S. Sudár and S. M. Qaim, Phys. Rev. C 53, 2885 (1996).

[45] S. Sudár and S. M. Qaim, Phys. Rev. C 73, 034613 (2006). 